\title{
Índices de seleção no aprimoramento simultâneo dos componentes da produção de frutos em açaizeiro
}

\author{
Davi Henrique Lima Teixeira(1), Maria do Socorro Padilha de Oliveira(2), \\ Flávia Maria Avelar Gonçalves ${ }^{(1)}$ e José Airton Rodrigues Nunes ${ }^{(1)}$
}

\begin{abstract}
(1)Universidade Federal de Lavras, Departamento de Biologia, Campus Universitário, Caixa Postal 3037, CEP $37200-000$ Lavras, MG. E-mail: davihlima@yahoo.com.br, avelar@ufla.br, jarnunes@dbi.ufla.br ${ }^{(2)}$ Embrapa Amazônia Oriental, Travessa Dr. Enéas Pinheiro, s/no, Caixa Postal 48, CEP 66095-100 Belém, PA. E-mail: spadilha@cpatu.embrapa.br
\end{abstract}

\begin{abstract}
Resumo - O objetivo deste trabalho foi determinar a estratégia seletiva mais adequada para o incremento simultâneo de componentes da produção de frutos em açaizeiro (Euterpe oleracea). Implantou-se um experimento com 25 progênies de meio-irmãos, no Município de Santa Izabel, PA, tendo-se utilizado o delineamento de blocos ao acaso, com quatro repetições e parcela de cinco plantas. Os índices de seleção de Smith \& Hazel, Williams, e Mulamba \& Mock foram aplicados em três estratégias seletivas. Foram utilizados, como pesos econômicos, os coeficientes de variação genéticos, a herdabilidade, a razão entre a correlação genética do caráter selecionado com a produção de frutos, e a somatória de todos os caracteres que compõem o índice e a produção de frutos. A ponderação pela razão das correlações permitiu que os índices de seleção discriminassem as melhores progênies nas diferentes estratégias de seleção avaliadas. A seleção simultânea por meio do índice de Mulamba \& Mock, quanto ao número de meses em frutificação, ao número de cachos colhidos, à produção de frutos e ao número de ráquilas no cacho, estima ganhos de forma mais robusta.
\end{abstract}

Termos para indexação: Euterpe oleracea, estratégia de seleção, resposta correlacionada, seleção de múltiplos caracteres.

\section{Selection index for simultaneously improving fruit production components of assai palm}

\begin{abstract}
The objective of this work was to determine the most appropriate strategy to simultaneously increase fruit production components of assai palm (Euterpe oleracea). An experiment with 25 half-sib progenies was carried out in the county of Santa Isabel, PA, Brazil, using a randomized complete block design with four replicates and five plants per plot. The Smith \& Hazel, Williams, and Mulamba \& Mock's selection indexes were applied in three selective strategies. The coefficients of genetic variation, heritability, the ratio between the genetic correlation of the trait under selection and fruit yield, and the sum of all genetic correlations between the traits that comprise the index and fruit yield were used as economic weights. Weighting of the correlation ratio allowed the selection indexes to discriminate the best progenies in the different selection strategies evaluated. Simultaneous selection by the Mulamba \& Mock index, as to the number of fruiting months, number of bunches harvested, fruit yield, and number of rachilles per bunch, robustly estimates genetic gains.
\end{abstract}

Index terms: Euterpe oleracea, selection strategy, correlated response, multiple traits selection.

\section{Introdução}

Tanto o fruto quanto o palmito têm importância econômica na exploração do açaizeiro (Euterpe oleracea Mart.). No entanto, com a notável expansão do mercado de frutos, os programas de melhoramento genético desta palmeira têm focado na seleção de progênies quanto a este caráter (Farias Neto et al., 2008; Oliveira \& Farias Neto, 2008). Contudo, a seleção não deve ser feita com base em apenas um caráter, uma vez que as cultivares de mérito econômico aliam caracteres de interesse do agricultor e do consumidor, ou seja, múltiplos caracteres (Bertini et al., 2010; Rodrigues et al., 2011).

No caso do açaizeiro, além da maior produção de frutos, também é interessante que se obtenham populações com menor entressafra, isto é, que produzam durante maior número de meses ao ano. Além disso, o menor tamanho de frutos é preferido pelos comerciantes, por apresentar maior rendimento da bebida "açaí" nas máquinas de processamento (Farias Neto et al., 2011).

A seleção simultânea de múltiplos caracteres pode ser realizada por diferentes métodos, e os índices de 
seleção têm sido frequentemente utilizados com este fim em fruteiras, como guaraná (Atroch et al., 2010), maracujazeiro-amarelo (Oliveira et al., 2008) e caju (Paiva et al., 2007). O primeiro índice de seleção proposto para a seleção de duas ou mais características correlacionadas foi o clássico (Smith, 1936; Hazel, 1943), com uso de variâncias e covariâncias genotípicas e fenotípicas. Porém, os grandes erros amostrais associados a estes parâmetros podem tornar esse índice menos efetivo em comparação a outros (Cruz \& Carneiro, 2006). Para contornar essas limitações, Williams (1962) propôs o índice base de seleção, no qual a combinação linear dos caracteres em seleção é feita pela ponderação direta dos valores fenotípicos com os respectivos pesos econômicos. O índice da soma de postos, proposto por Mulamba \& Mock (1978), apresenta a vantagem de não necessitar de pesos econômicos nem da estimação de parâmetros além das médias. Esse índice é obtido após o ordenamento dos genótipos quanto ao caráter avaliado no sentido desejado, e posterior somatório das suas classificações.

Tanto o índice clássico quanto o índice base preconizam a atribuição de pesos econômicos na sua construção. Na proposta inicial, esses pesos deveriam ser obtidos em função do valor de mercado de cada caráter por unidade avaliada (Smith, 1936; Hazel, 1943; Williams, 1962). Contudo, valores de mercado mudam constantemente e, para alguns caracteres, não há como encontrar um valor econômico. Portanto, na literatura, tem-se atribuído, como peso econômico, estimativas de parâmetros genéticos referentes à população em estudo, como herdabilidade e coeficiente de variação genético, além de pesos por tentativas para obter predições de ganhos favoráveis ao melhoramento (Granate et al., 2002; Gonçalves et al., 2007; Cândido et al., 2011). De acordo com Resende (2002), melhores predições de ganhos podem ser obtidas por índices de seleção com pesos econômicos que considerem as estruturas de correlação entre os caracteres selecionados.

As correlações podem contornar dificuldades no melhoramento de caracteres que apresentem problemas na mensuração em virtude do efeito pronunciado de ambiente, ou que apresentem baixa herdabilidade, por permitir a seleção de caracteres correlacionados que não apresentem esses problemas (Falconer \& Mackay, 1996). Cabe ao melhorista verificar quais caracteres devem participar do índice de seleção para predizer melhores ganhos, em grandeza e sentido, para os caracteres em avaliação, bem como associar a seleção direta e indireta para o incremento nos ganhos do caráter de maior interesse.

O objetivo deste trabalho foi determinar a estratégia seletiva mais adequada para o incremento simultâneo de componentes da produção de frutos em açaizeiro.

\section{Material e Métodos}

Foram avaliados caracteres relativos à produção de frutos e de cacho em progênies de meio-irmãos de açaizeiro, nos anos de 2005, 2006 e 2007, selecionadas no Banco de Germoplasma da Embrapa Amazônia Oriental como promissoras quanto à produção de frutos. Essas progênies foram instaladas em fevereiro de 2001, em área de produtor rural no Município de Santa Izabel, PA, pertencente à mesorregião metropolitana de Belém. O clima é predominantemente equatorial quente e úmido, do tipo $\mathrm{Af}_{1}$, conforme a classificação de Köppen, com temperatura média de $25^{\circ} \mathrm{C}$, altitude de $24 \mathrm{~m}$ e a maior precipitação pluvial do Estado do Pará: acima de $3.000 \mathrm{~mm}$ por ano.

Utilizou-se o delineamento experimental de blocos ao acaso, com 25 progênies, quatro repetições e parcelas lineares de cinco plantas. $\mathrm{O}$ espaçamento utilizado no plantio foi de $6 \times 4 \mathrm{~m}$. Foram utilizadas bordaduras constituídas por plantas das 25 progênies misturadas. Três fileiras de bordadura foram colocadas no início e no final do experimento, e duas fileiras nas laterais e entre os blocos.

Os caracteres mensurados foram: número total de meses em produção (NMP), obtido pela contagem do número total de meses em que houve a coleta de cacho em cada planta ao ano; número total de cachos colhidos (NTC), determinado pela contagem do número de cachos coletados por planta ao ano; produção total de frutos (PTF), obtida pela soma dos pesos dos frutos produzidos em cada planta ao ano (quilograma); peso total do cacho (PTC), calculado pela média dos pesos (quilograma) dos cachos completos (frutos mais raque e ráquilas); peso de frutos por cacho $(\mathrm{PFC})$, obtido pela média dos pesos dos frutos dos cachos (quilograma); rendimento de frutos por cacho (RFC), determinado pela razão entre peso de frutos por cacho e peso total do cacho, multiplicado por 100 (percentagem); número de ráquilas do cacho (NRC), obtido pela média da contagem das ráquilas de cada cacho por planta ao ano; 
comprimento da raque do cacho (CRC), mensurado com fita métrica a partir da inserção da raque no pecíolo até o final da raque (centímetros); e peso de cem frutos (PCF), obtido pela média do peso de cem frutos, retirados aleatoriamente dos cachos (gramas).

Os dados referentes aos três anos de avaliação foram submetidos à análise de variância conjunta, tendo-se observado seus pressupostos, de acordo com o modelo estatístico $\mathrm{y}_{\mathrm{ijq}}=\mathrm{m}+\mathrm{b}_{\mathrm{j}}+\mathrm{p}_{\mathrm{i}}+\mathrm{a}_{\mathrm{q}}+(\mathrm{bp})_{\mathrm{ij}}+(\mathrm{ba})_{\mathrm{jq}}+$ $(\mathrm{pa})_{\mathrm{iq}}+\varepsilon_{\mathrm{ijq}}$, em que: $\mathrm{y}_{\mathrm{ijq}}$ é a observação da progênie $\mathrm{i}$ no bloco $\mathrm{j}$ no ano $\mathrm{q}$; $\mathrm{m}$ é a constante associada às observações; $b_{j}$ é o efeito aleatório do bloco $j$, sendo $\mathrm{b} \sim \mathrm{N}\left(0, \sigma_{\mathrm{b}}^{2}\right) ; \mathrm{p}_{\mathrm{i}}$ é o efeito aleatório da progênie $\mathrm{i}$, sendo $\mathrm{p} \sim \mathrm{N}\left(0, \sigma_{\mathrm{p}}^{2}\right) ; \mathrm{a}_{\mathrm{q}}$ é o efeito fixo do ano q; $(\mathrm{bp})_{\mathrm{ij}}$ é o efeito aleatório da interação entre o bloco $\mathrm{j}$ e a progênie i, sendo bp $\sim \mathrm{N}\left(0, \sigma_{\mathrm{bp}}^{2}\right)$; (ba) $)_{\mathrm{jq}}$ é o efeito aleatório da interação entre o bloco j e o ano q, sendo ba $\sim \mathrm{N}\left(0, \sigma_{\mathrm{ba}}^{2}\right)$; (pa) iq é o efeito aleatório da interação entre a progênie i e o ano q, sendo pa N $\left(0, \sigma_{\mathrm{pa}}^{2}\right)$; e $\varepsilon_{\mathrm{ijq}}$ é o efeito aleatório do erro experimental, sendo e $\sim \mathrm{N}\left(0 ; \sigma_{\mathrm{e}}^{2}\right)$.

A partir das esperanças dos quadrados médios, foram estimados os componentes de variância associados aos efeitos aleatórios do modelo. Para cada caráter, foram obtidas as estimativas da herdabilidade na média de progênies, do coeficiente de variação genético, da acurácia seletiva (Resende \& Duarte, 2007) e das correlações genéticas entre os caracteres. Os erros-padrão das correlações genéticas foram obtidos por procedimento análogo ao apresentado por Holland (2006). Os limites superiores e inferiores da herdabilidade foram estimados de acordo com Knapp et al. (1985).

Para a construção dos índices de seleção, foram consideradas três estratégias seletivas, que variaram em função dos caracteres envolvidos. Assim, realizou-se seleção simultânea quanto aos caracteres: estratégia 1, produção de frutos, número de meses em produção e de cachos colhidos, peso de frutos por cacho e número de ráquilas do cacho; estratégia 2, produção de frutos e número de meses em produção, de cachos colhidos e de ráquilas no cacho; e estratégia 3, produção de frutos, número de cachos colhidos e peso de frutos por cacho.

Os índices de seleção avaliados foram o índice clássico de Smith (1936) e Hazel (1943), o índice base de Williams (1962) e o índice da soma de postos de Mulamba \& Mock (1978).
O índice clássico (Smith \& Hazel) foi obtido pelo seguinte estimador:

$$
\mathrm{I}_{\mathrm{i}}=\sum_{\mathrm{k}} \mathrm{b}_{\mathrm{k}} \overline{\mathrm{y}}_{\mathrm{ik}}
$$

em que: $I_{i}$ é o valor do índice calculado para a progênie $i ; b_{k}$ é o coeficiente de ponderação do índice associado ao caráter $\mathrm{k}$; e $\bar{y}_{\mathrm{ik}}$ é a média fenotípica da progênie i relativa ao caráter $k$. Os valores de $b_{k}$ foram estimados por $b=\mathrm{P}^{-1} \mathrm{G} \times$ a, em que: $\mathrm{P}^{-1}$ é a inversa da matriz de covariâncias fenotípicas médias entre caracteres; $\mathrm{G}$ é a matriz das variâncias e covariâncias genotípicas na média de progênies entre os caracteres; e a é o vetor dos pesos econômicos dos caracteres.

O índice base (Williams, 1962) foi obtido pelo estimador

$$
\mathrm{I}_{\mathrm{i}}=\sum_{\mathrm{k}} \mathrm{b}_{\mathrm{k}} \mathrm{a} \overline{\mathrm{y}}_{\mathrm{ik}} .
$$

Já o índice de soma de postos de Mulamba \& Mock (1978), foi calculado pela expressão

$$
\mathrm{I}_{\mathrm{i}}=\sum_{\mathrm{k}} \mathrm{ar}_{\mathrm{ik}}
$$

em que: $\mathrm{r}_{\mathrm{ik}}$ é o posto da progênie i para o caráter k.

Os pesos econômicos utilizados nos três índices foram o coeficiente de variação genético, a herdabilidade e a razão da correlação genética entre o caráter selecionado e a produção de frutos, por meio da somatória de todas as correlações dos caracteres que compõem o índice e a produção de frutos, conforme Resende (2002).

Para cada caráter, foi estimada a resposta à seleção pelos índices, de acordo com Cruz \& Carneiro (2006).

Todas as análises foram realizadas com uso do programa Genes (Cruz, 2006).

\section{Resultados e Discussão}

Entre os caracteres avaliados, apenas a produção de frutos apresentou baixa precisão experimental (Tabela 1), evidenciada pela magnitude da acurácia seletiva (Resende \& Duarte, 2007). Os demais caracteres foram de moderada (rendimento de frutos por cacho), alta (número de meses em produção e de cachos colhidos, e peso total do cacho e de frutos por cacho) a muito alta precisão experimental (número de ráquilas do cacho, comprimento da raque do cacho e peso de cem frutos). Esse resultado está de acordo com 
a significância da variação genética entre progênies, em que, dos nove caracteres avaliados, oito foram significativos pelo teste F. Portanto, a população avaliada mostra-se promissora para a obtenção de ganho genético por meio da seleção, para a maioria dos caracteres em estudo. Por apresentar estimativas de herdabilidade elevada, destacaram-se os caracteres: número de ráquilas do cacho, comprimento da raque do cacho e peso de cem frutos.

Na cultura do açaizeiro, a produção de frutos é um dos principais caracteres que é objetivo de melhoramento. Contudo, para as progênies avaliadas, a estimativa de herdabilidade foi de baixa magnitude $(21,37 \%)$, conforme a classificação proposta por Resende (2002). Assim, esperam-se dificuldades no ganho genético por meio da seleção direta para esse caráter. Esse resultado foi inferior ao obtido por Farias Neto et al. (2008), que, ao avaliar uma safra de progênies de polinização aberta de açaizeiro irrigado, observaram estimativa de herdabilidade de $54,82 \%$. A produção de frutos é um caráter complexo e muito influenciado pelo ambiente, cuja expressão fenotípica é função da expressão de vários outros caracteres (componentes de produção), como peso do fruto e número de cachos e de frutos por cacho, o que pode justificar o uso da seleção indireta para a produção de frutos (Oliveira et al., 2000).

De acordo com Falconer \& Mackay (1996), a resposta correlacionada é vantajosa quando o produto da correlação entre dois caracteres e a herdabilidade do caráter ao qual a seleção foi aplicada for maior que a herdabilidade do caráter no qual se deseja obter ganhos. Verificou-se que esta condição ocorreu para as correlações da produção de frutos com o número de ráquilas do cacho, e de meses em produção com o número de cachos colhidos e com o peso de cem frutos (Tabelas 1 e 2 ). Constatou-se que alguns

Tabela 1. Parâmetros genéticos e fenotípicos para número total de meses em produção (NMP), número total de cachos (NTC), número de ráquilas do cacho (NRC), produção total de frutos (PTF), peso total do cacho (PTC), peso de frutos por cacho (PFC), rendimento de frutos por cacho (RFC), comprimento da raque do cacho (CRC) e peso de cem frutos (PCF), obtidos pela análise conjunta de três anos de produção de frutos, em progênies de meio-irmãos de açaizeiro.

\begin{tabular}{|c|c|c|c|c|c|c|c|c|c|}
\hline Parâmetro $^{(1)}$ & NMP & NTC & $\mathrm{NRC}$ & $\begin{array}{l}\text { PTF } \\
------- \\
\end{array}$ & $\begin{array}{c}\text { PTC } \\
--(\mathrm{kg})-- \\
\end{array}$ & PFC & $\begin{array}{c}\text { RFC } \\
(\%)\end{array}$ & $\begin{array}{l}\text { CRC } \\
(\mathrm{cm})\end{array}$ & $\begin{array}{c}\text { PCF } \\
(\mathrm{g})\end{array}$ \\
\hline$\sigma_{\mathrm{p}}^{2}$ & $0,07 * *$ & $0,34 * *$ & $32,96 * *$ & $0,32 \mathrm{~ns}$ & $0,06 * *$ & $0,03 * *$ & $2,12 *$ & $7,24 * *$ & $167,38 * *$ \\
\hline$\sigma_{\mathrm{pa}}^{2}$ & $0,51^{\mathrm{ns}}$ & $0,09^{\mathrm{ns}}$ & $2,77^{*}$ & $0,70 *$ & $0,05 * *$ & $0,03 * *$ & $0,59^{\mathrm{ns}}$ & $0,92 *$ & $8,72 *$ \\
\hline$h^{2}(\%)$ & 55,10 & 55,49 & 86,52 & 21,37 & 65,17 & 54,66 & 43,80 & 80,85 & 87,65 \\
\hline $\mathrm{LI}^{2}(\%)$ & 7,64 & 8,44 & 72,27 & 61,74 & 28,35 & 6,73 & 15,60 & 60,61 & 74,60 \\
\hline $\mathrm{LS} \mathrm{h}^{2}(\%)$ & 75,61 & 75,82 & 92,68 & 57,28 & 81,08 & 75,37 & 69,47 & 89,60 & 93,29 \\
\hline $\mathrm{CV}_{\mathrm{g}}$ & 11,95 & 13,38 & 6,42 & 6,43 & 9,48 & 9,03 & 2,12 & 5,65 & 8,70 \\
\hline$\hat{\mathrm{r}}_{\mathrm{g} \hat{\mathrm{g}}}$ & 0,74 & 0,75 & 0,93 & 0,46 & 0,81 & 0,74 & 0,66 & 0,90 & 0,94 \\
\hline Média & 2,29 & 4,38 & 89,48 & 8,80 & 2,50 & 1,77 & 68,68 & 40,61 & 148,77 \\
\hline
\end{tabular}

${ }^{(1)} \sigma_{\mathrm{p}}^{2}$, variância genética entre progênies; $\sigma_{\mathrm{pa}}^{2}$, variância da interação progênie x ano; $h^{2}$, herdabilidade no sentido restrito para média de progênies; LI e LS, limite inferior e superior, respectivamente; $\mathrm{CV}_{\mathrm{g}}$, coeficiente de variação genético entre progênies; e $\mathrm{r}_{\mathrm{g} \hat{\mathrm{g}}}$, acurácia seletiva. ${ }^{\mathrm{n}} \mathrm{Não}$ significativo. ${ }^{*} \mathrm{e}{ }^{*} *$ Significativo a 5 e $1 \%$ de probabilidade.

Tabela 2. Estimativas das correlações genéticas \pm erro padrão entre nove caracteres relativos à produção de frutos e ao cacho, em progênies de meio-irmãos de açaizeiro, obtidas pela análise conjunta dos anos de 2005, 2006 e 2007, na média de parcela.

\begin{tabular}{|c|c|c|c|c|c|c|c|c|c|}
\hline Caráter(1) & NMP & NTC & PTF & PTC & PFC & $\mathrm{RFC}$ & NRC & $\mathrm{CRC}$ & PCF \\
\hline NMP & - & $0,93 * \pm 0,06$ & $0,69 * \pm 0,31$ & $-0,58 \pm 0,37$ & $-0,45 \pm 0,41$ & $0,05 \pm 0,36$ & $-0,29 \pm 0,27$ & $-0,30 \pm 0,30$ & $0,08 \pm 0,07$ \\
\hline NTC & & - & $0,58 * \pm 0,27$ & $-0,61 \pm 0,33$ & $-0,57 \pm 0,36$ & $-0,01 \pm 0,32$ & $-0,25 \pm 0,25$ & $-0,41 \pm 0,24$ & $-0,13 \pm 0,25$ \\
\hline PTF & & & - & $0,19 \pm 0,48$ & $0,34 \pm 0,36$ & $-0,07 \pm 0,46$ & $0,46 \pm 0,30$ & $-0,18 \pm 0,41$ & $-0,29 \pm 0,37$ \\
\hline PTC & & & & - & $0,93 * \pm 0,05$ & $-0,49 \pm 0,43$ & $0,98 * \pm 0,11$ & $0,58 * \pm 0,21$ & $0,02 \pm 0,30$ \\
\hline $\mathrm{PFC}$ & & & & & - & $-0,13 \pm 0,45$ & $0,82 * \pm 0,17$ & $0,41 \pm 0,27$ & $0,01 \pm 0,07$ \\
\hline RFC & & & & & & - & $-0,60 * \pm 0,22$ & $-0,71 * \pm 0,22$ & $-0,07 \pm 0,27$ \\
\hline NRC & & & & & & & - & $0,57 * \pm 0,16$ & $-0,06 \pm 0,22$ \\
\hline $\mathrm{CRC}$ & & & & & & & & - & $0,35 \pm 0,20$ \\
\hline $\mathrm{PCF}$ & & & & & & & & & - \\
\hline
\end{tabular}

${ }^{(1)}$ NMP, número total de meses em produção; NTC, número total de cachos emitidos; PTF, produção total de frutos; PTC, peso total do cacho; PFC, peso de frutos por cacho; RFC, rendimento de frutos por cacho; NRC, número de ráquilas do cacho; CRC, comprimento da raque do cacho; e PCF, peso de cem frutos. *Significativo a 5\% de probabilidade pelo teste t de Student, nos casos em que o erro padrão é, ao menos, 50\% inferior a sua estimativa (Steel et al., 1997). 
pares de caracteres apresentaram correlação genética significativa, como, por exemplo: produção de frutos com número de cachos colhidos e de meses em produção (Tabela 2).

Para obter ganhos indiretos na produção de frutos, foram selecionados, simultaneamente, os caracteres de maior correlação com esse caráter (Tabela 3). Por meio da seleção simultânea da produção de frutos, do número de meses em produção e de cachos colhidos, do peso de frutos por cacho e do número de ráquilas do cacho, o índice de Smith \& Hazel permitiu pequenos ganhos na produção de frutos, quando ponderado pelo coeficiente de variação genético $\left(\mathrm{CV}_{\mathrm{g}}\right)$. Esse índice estimou ganhos positivos e elevados para o peso total do cacho e de frutos por cacho, quando os pesos econômicos aplicados foram o $\mathrm{CV}_{\mathrm{g}}$ e a herdabilidade na média de progênies $\left(h^{2}\right)$. Entretanto, os ganhos preditos para o número de meses em produção e de cachos colhidos foram baixos. Cabe ressaltar que o número de meses em produção é tão importante quanto a produção de frutos, já que a entressafra, que se estende de janeiro a julho, é o principal obstáculo para a exportação de frutos do açaizeiro (Santana et al., 2008).

O índice de Williams, ou índice base, apresentou resultado superior ao índice clássico, quando foram utilizadas, como peso econômico, a $\mathrm{h}^{2}$ dos caracteres e a razão das correlações genéticas (RC) (Tabela 3). $\mathrm{O}$ índice de Mulamba \& Mock foi superior ao índice base quando os pesos foram o $\mathrm{CV}_{\mathrm{g}}$ e a RC. Amaral Junior et al. (2010) verificaram melhores resultados com o uso do índice da soma de postos, em comparação ao clássico, em populações de milho-pipoca originadas de quatro ciclos de seleção recorrente. Freitas Júnior et al. (2009) também relataram superioridade do índice da soma de postos quando comparado ao clássico, ao índice base, e a outros índices, na seleção simultânea para 12 caracteres avaliados em milho-pipoca.

Pelo índice da soma de postos, com o uso do $\mathrm{CV}_{\mathrm{g}} \mathrm{e}$ da RC como pesos, foram obtidos ganhos satisfatórios e mais bem distribuídos em relação aos caracteres de maior importância, como produção de frutos, peso de frutos por cacho e número de cachos e de meses em produção (Tabela 3). Além disso, esse índice permitiu estimar ganhos em sentido favorável para peso de cem frutos, uma vez que os comerciantes de açaí preferem frutos de tamanho reduzido, por apresentarem maior rendimento de polpa. Para o índice base, a ponderação pelo $\mathrm{CV}_{\mathrm{g}}$ estimou ganhos negativos para a maioria dos caracteres. Arnhold \& Silva (2009) também constataram que o índice de Mulamba \& Mock apresenta melhor distribuição dos ganhos, em sentido favorável para capacidade de expansão, rendimento de grãos e altura de plantas, em milho-pipoca.

Tabela 3. Estimativas de ganhos genéticos percentuais, pelos índices de Smith \& Hazel, Williams e Mulamba \& Mock, com uso de diferentes pesos econômicos aplicados na seleção simultânea de caracteres, por meio das estratégias 1,2 e 3, em progênies de meio-irmãos de açaizeiro.

\begin{tabular}{|c|c|c|c|c|c|c|c|c|c|}
\hline \multirow[t]{2}{*}{ Caráter $^{(1)}$} & \multicolumn{3}{|c|}{ Smith \& Hazel } & \multicolumn{3}{|c|}{ Williams } & \multicolumn{3}{|c|}{ Mulamba \& Mock } \\
\hline & $\mathrm{CV}_{\mathrm{g}}$ & $\mathrm{h}^{2}$ & $\mathrm{RC}$ & $\mathrm{CV}_{\mathrm{g}}$ & $\mathrm{h}^{2}$ & $\mathrm{RC}$ & $\mathrm{CV}_{\mathrm{g}}$ & $h^{2}$ & $\mathrm{RC}$ \\
\hline & \multicolumn{9}{|c|}{ Estratégia $^{(2)} 1$} \\
\hline NMP & 1,90 & $-1,47$ & 1,90 & $-3,38$ & 3,18 & 3,18 & 7,04 & 5,43 & 7,04 \\
\hline NTC & 3,62 & 0,84 & 3,62 & $-2,27$ & 3,50 & 3,50 & 7,80 & 5,82 & 7,80 \\
\hline PTF & 3,18 & 2,64 & 3,18 & $-1,24$ & 3,84 & 3,84 & 3,99 & 4,01 & 3,99 \\
\hline PTC & 10,19 & 8,86 & 10,19 & $-5,53$ & 9,49 & 9,49 & 7,28 & 9,44 & 7,28 \\
\hline $\mathrm{PFC}$ & 8,10 & 7,22 & 8,10 & $-3,53$ & 7,97 & 7,97 & 6,16 & 7,77 & 6,16 \\
\hline RFC & $-0,27$ & $-0,06$ & $-0,27$ & 0,86 & $-0,08$ & $-0,08$ & $-0,13$ & $-0,30$ & $-0,13$ \\
\hline NRC & 8,06 & 8,48 & 8,06 & $-6,16$ & 8,27 & 8,27 & 6,51 & 7,15 & 6,51 \\
\hline CRC & 4,76 & 3,81 & 4,76 & $-1,78$ & 4,59 & 4,59 & 3,23 & 4,97 & 3,23 \\
\hline \multirow[t]{2}{*}{ PCF } & $-0,66$ & $-2,18$ & $-0,66$ & $-1,89$ & 1,14 & 1,14 & $-0,88$ & 1,83 & $-0,88$ \\
\hline & \multicolumn{9}{|c|}{ Estratégia 2} \\
\hline NMP & $-1,47$ & $-1,47$ & 3,18 & 3,18 & 3,18 & 3,18 & 10,17 & 7,04 & 7,04 \\
\hline NTC & 0,84 & 0,84 & 3,50 & 3,50 & 3,50 & 3,50 & 11,17 & 7,80 & 7,80 \\
\hline PTF & 2,64 & 2,64 & 3,84 & 3,84 & 3,84 & 3,84 & 1,84 & 3,99 & 3,99 \\
\hline PTC & 8,86 & 8,86 & 9,49 & 9,49 & 9,49 & 9,49 & $-0,59$ & 7,28 & 7,28 \\
\hline PFC & 7,22 & 7,22 & 7,97 & 7,97 & 7,97 & 7,97 & $-1,29$ & 6,16 & 6,16 \\
\hline $\mathrm{RFC}$ & $-0,06$ & $-0,06$ & $-0,08$ & $-0,08$ & $-0,08$ & $-0,08$ & $-0,83$ & $-0,13$ & $-0,13$ \\
\hline NRC & 8,48 & 8,48 & 8,27 & 8,27 & 8,27 & 8,27 & 1,69 & 6,51 & 6,51 \\
\hline $\mathrm{CRC}$ & 3,81 & 3,81 & 4,59 & 4,59 & 4,59 & 4,59 & 1,98 & 3,23 & 3,23 \\
\hline \multirow[t]{2}{*}{ PCF } & $-2,18$ & 2,18 & 1,14 & 1,14 & 1,14 & 1,14 & $-0,23$ & $-0,88$ & $-0,88$ \\
\hline & \multicolumn{9}{|c|}{ Estratégia 3} \\
\hline NMP & 9,69 & 9,69 & 7,44 & 7,60 & 7,04 & 5,75 & 5,43 & 5,43 & 5,43 \\
\hline NTC & 12,06 & 12,06 & 9,2 & 10,28 & 7,80 & 6,71 & 5,82 & 5,82 & 5,82 \\
\hline PTF & 1,00 & 1,00 & 2,04 & 3,69 & 3,99 & 4,28 & 4,01 & 4,01 & 4,01 \\
\hline PTC & $-2,17$ & $-2,17$ & 3,69 & 1,79 & 7,28 & 5,88 & 9,44 & 9,44 & 9,44 \\
\hline PFC & $-2,74$ & $-2,74$ & 2,56 & 1,45 & 6,16 & 5,47 & 7,77 & 7,77 & 7,77 \\
\hline RFC & $-0,63$ & $-0,63$ & $-0,24$ & $-0,22$ & $-0,13$ & 0,20 & $-0,30$ & $-0,30$ & $-0,30$ \\
\hline NRC & $-0,31$ & $-0,31$ & 2,43 & 3,35 & 6,51 & 4,69 & 7,15 & 7,15 & 7,15 \\
\hline CRC & 0,98 & 0,98 & 2,42 & 0,59 & 3,23 & 3,08 & 4,97 & 4,97 & 4,97 \\
\hline PCF & $-1,05$ & $-1,05$ & 0,46 & $-3,98$ & $-0,88$ & $-1,34$ & 1,83 & 1,83 & 1,83 \\
\hline
\end{tabular}

(1)NMP, número total de meses em produção; NTC, número total de cachos; PTF, produção total de frutos; PTC, peso total do cacho; PFC, peso de frutos por cacho; RFC, rendimento de frutos por cacho; NRC, número de ráquilas do cacho; CRC, comprimento da raque do cacho; e PCF, peso de cem frutos. ${ }^{(2)}$ Estratégia 1, seleção simultânea quanto aos caracteres PFT, NMP, NTC, PFC e NRC; estratégia 2, seleção simultânea quanto aos caracteres PTF, NMP, NTC e NRC; estratégia 3, seleção simultânea quanto aos caracteres PTF, NTC e PFC. $\mathrm{CV}_{\mathrm{g}}$, coeficiente de variação genético; $\mathrm{h}^{2}$, herdabilidade na média de progênies; RC, razão entre a correlação genética do caráter sob seleção com o caráter principal, e somatória de todas as correlações entre os caracteres que compõem o índice, com o caráter principal. Para esta última ponderação, os pesos econômicos dos caracteres em seleção em cada estratégia seletiva são: estratégia 1, NMP, 0,289916, NTC, 0,232932, PFT, 0,483092, PFC, 0,124542 e NRC, 0,176245; estratégia 2, NMP, 0,338235, NTC, 0,269767, PTF, 0,578035 e NRC, 0,202643; estratégia 3, NMP, 0,436709 , NTC, 0,343195 e PTF, 0,787402 . 
$\mathrm{Na}$ segunda estratégia de seleção, o caráter peso de frutos por cacho foi retirado, em razão de ter apresentado menor correlação com a produção de frutos e menor herdabilidade. Por meio dessa estratégia, o índice da soma de postos, ponderado pela $\mathrm{h}^{2}$ e pela $\mathrm{RC}$, estimou ganhos preditos equivalentes quando o peso de frutos por cacho foi considerado no cálculo do índice ponderado pelo $\mathrm{CV}_{\mathrm{g}}$ (Tabela 3 ).

Como busca descrever a associação por meio da variação conjunta das características em estudo, a correlação pode ser subestimada se uma das variáveis apresentar pouca variação (Montardo et al., 2003). Por esse motivo, utilizou-se uma terceira estratégia de seleção com base nos caracteres com maiores efeitos diretos ou com maior contribuição para a produção de frutos: número total de cachos emitidos e peso de frutos por cacho. De acordo com Oliveira et al. (2000), esses caracteres explicam $92 \%$ da variação na produção de frutos e são seus principais determinantes.

Com essa última estratégia seletiva, o índice base apresentou os mesmos ganhos preditos que as duas primeiras estratégias, pelo índice da soma de postos (Tabela 3). Contudo, vale ressaltar que, nessa seleção simultânea, não foram considerados caracteres pouco influenciados pelo ambiente, como número de ráquilas do cacho, o que torna essa estratégia menos robusta. Segundo Pedrozo et al. (2009), um índice pode ser mais adequado que outro em uma situação, e o contrário pode ocorrer em outra. A eficiência de seleção pelos índices utilizados foi inerente à população em estudo, uma vez que, entre outros fatores, foram consideradas como pesos as estimativas de parâmetros genéticos que variam de acordo com a população e com o controle experimental realizado.

Ao se considerar os ganhos estimados com as estratégias seletivas, os índices de seleção e os pesos econômicos utilizados, verifica-se que a segunda estratégia seletiva é a mais interessante, pois inclui o caráter número de ráquilas do cacho, que apresenta alta herdabilidade, o que torna a seleção dos melhores genótipos, com base nos fenótipos, mais segura. Com o uso dessa estratégia, o índice de Mulamba \& Mock, ponderado tanto pela herdabilidade dos caracteres quanto pela razão das correlações, proporcionou estimativas razoáveis para os principais componentes da produção de frutos do açaizeiro: peso de frutos por cacho e número de meses em produção e de cachos colhidos. Quanto aos pesos econômicos, a razão entre as correlações permitiu que os índices proporcionassem as melhores estimativas de ganhos com a seleção, na maioria das situações.

\section{Conclusões}

1. Um peso econômico que considera as estruturas de correlação entre os caracteres selecionados pelo índice discrimina melhor as progênies de açaizeiro mais produtivas.

2. O índice de Mulamba \& Mock é mais eficiente para estimar ganhos nos componentes da produção de frutos de açaizeiro.

\section{Referências}

AMARAL JUNIOR, A.T.; FREITAS JUNIOR, S.P.; RANGEL, R.M.;PENA, G.F.; RIBEIRO, R.M.;MORAIS, R.C.; SCHUELTER, A.R. Improvement of a popcorn population using selection indexes from a fourth cycle of recurrent selection program carried out in two different environments. Genetics and Molecular Research, v.9, p.340-347, 2010.

ARNHOLD, E.; SILVA, R.G. Eficiências relativas de índices de seleção considerando espécies vegetais e pesos econômicos iguais entre caracteres. Bioscience Journal, v.25, p.76-82, 2009.

ATROCH, A.L.; NASCIMENTO FILHO, F.J. do; RESENDE, M.D.V. de; LOPES, R.; CLEMENT, C.R. Avaliação e seleção de progênies de meios-irmãos de guaranazeiro. Revista de Ciências Agrárias, v.53, p.123-130, 2010.

BERTINI, C.H.C. de M.; ALMEIDA, W.S. de; SILVA, A.P.M. da; SILVA, J.W.L. e; TEÓFILO, E.M. Análise multivariada e índice de seleção na identificação de genótipos superiores de feijão-caupi. Acta Scientiarum. Agronomy, v.32, p.613-619, 2010

CANDIDO, L.S.; ANDRADE. J.A. da C.; GARCIA, F.Q.; GONÇALVES, L.S.A.; AMARAL JÚNIOR, A.T. do. Seleção de progênies de meios-irmãos do composto Isanão VF-1 de milho na safra e safrinha. Ciência Rural, v.41, p.947-953, 2011.

CRUZ, C.D. Programa GENES: biometria. Viçosa: UFV, 2006. 382p.

CRUZ, C.D.; CARNEIRO, P.C.S. Modelos biométricos aplicados ao melhoramento genético. 2.ed. Viçosa: UFV, 2006. v.2, 585p.

FALCONER, D.S.; MACKAY, T.F.C. Introduction to quantitative genetics. London: Longman Malaysia, 1996. 463p.

FARIAS NETO, J.T. de; RESENDE, M.D.V. de; OLIVEIRA, M. do S.P. de; NOGUEIRA, O.L.; FALCÃO, P.N.B.; SANTOS, N.S.A. dos. Estimativas de parâmetros genéticos e ganhos de seleção em progênies de polinização aberta de açaizeiro. Revista Brasileira de Fruticultura, v.30, p.1051-1056, 2008.

FARIAS NETO, J.T. de; RESENDE, M.D.V. de; OLIVEIRA, M. do S.P. de. Seleção simultânea em progênies de açaizeiro irrigado para produção e peso de fruto. Revista Brasileira de Fruticultura, v.33, p.532-539, 2011 
FREITAS JÚNIOR, S. de P.; AMARAL JUNIOR, A.T. do; RANGEL, R.M.; VIANA, A.P. Predição de ganhos genéticos na população de milho pipoca UNB-2U sob seleção recorrente utilizando-se diferentes índices de seleção. Semina: Ciências Agrárias, v.30, p.803-814, 2009.

GONÇALVES, G.M.; VIANA, A.P.; BEZERRA NETO, F.V.; PEREIRA, M.G.; PEREIRA, T.N.S. Seleção e herdabilidade na predição de ganhos genéticos em maracujá-amarelo. Pesquisa Agropecuária Brasileira, v.42, p.193-198, 2007.

GRANATE, M.J.; CRUZ, C.D.; PACHECO, C.A.P. Predição de ganho genético com diferentes índices de seleção no milho pipoca CMS-43. Pesquisa Agropecuária Brasileira, v.37, p.1001-1008, 2002.

HAZEL, L.N. The genetics basics for constructing selections indexes. Genetics, v.28, p.476-490, 1943.

HOLLAND, J.B. Estimating genotypic correlations and their standard errors using multivariate restricted maximum likelihood estimation with SAS Proc MIXED. Crop Science, v.46, p.642-654, 2006.

KNAPP, S.J.; STROUP, W.W.; ROSS, W.M. Exact confidence intervals for heritability on a progeny mean basis. Crop Science, v.25, p.192-194, 1985.

MONTARDO, D.P.; DALL'AGNOL, M.; CRUSIUS, A.F.; PAIM, N.R. Análise de trilha para rendimento de sementes em trevo vermelho (Trifolium pratense L.). Revista Brasileira de Zootecnia, v.32, p.1076-1082, 2003.

MULAMBA, N.N.; MOCK, J.J. Improvement of yield potential of the Eto Blanco maize (Zea mays L.) population by breeding for plant traits. Egypt Journal of Genetic and Cytology, v.7, p.40-51, 1978.

OLIVEIRA, E.J.de; SANTOS, V.daS; LIMA, D.S. de;MACHADO, M.D.; LUCENA, R.S.; MOTTA, T.B.N; CASTELLEN, M. da S. Seleção em progênies de maracujazeiro-amarelo com base em índices multivariados. Pesquisa Agropecuária Brasileira, v.43, p.1543-1549, 2008.

OLIVEIRA, M. do S.P. de; FARIAS NETO, J.T. de. Seleção massal em açaizeiros para a produção de frutos. Revista de Ciências Agrárias, n.49, p.145-156, 2008.
OLIVEIRA, M. do S.P. de; LEMOS, M.A.; SANTOS, V.F. dos; SANTOS, E.O. dos. Coeficiente de caminhamento entre caracteres agronômicos e a produção de frutos em açaizeiro (Euterpe oleracea Mart.). Revista Brasileira de Fruticultura, v.22, p.6-10, 2000.

PAIVA, J.R. de; CAVALCANTI, J.J.V.; BARROS, L. de M.; CORRÊA, M.C. de M.; MAIA, M.C.C.; COSTA FILHO, A.B. Seleção de clones de cajueiro comum pelo método em "Tandem" e índice de classificação. Ciência e Agrotecnologia, v.31, p.765-772, 2007.

PEDROZO, C.Â.; BENITES, F.R.G.; BARBOSA, M.H.P.; RESENDE, M.D.V. de; SILVA, F.L. da. Eficiência de índices de seleção utilizando a metodologia REML/BLUP no melhoramento da cana-de-açúcar. Scientia Agraria, v.10, p.31-36, 2009.

RESENDE, M.D.V. de. Genética biométrica e estatística no melhoramento de plantas perenes. Brasília: Embrapa Informação Tecnológica; Colombo: Embrapa Florestas, 2002. 975p.

RESENDE, M.D.V. de; DUARTE, J.B. Precisão e controle de qualidade em experimentos de avaliação de cultivares. Pesquisa Agropecuária Tropical, v.37, p.182-194, 2007.

RODRIGUES, F.; VON PINHO, R.G.; ALBUQUERQUE, C.J.B.; VON PINHO, E.V.R. Índice de seleção e estimativa de parâmetros genéticos e fenotípicos para características relacionadas com a produção de milho-verde. Ciência e Agrotecnologia, v.35, p.278-286, 2011.

SANTANA, A.C. de; CARVALHO, D.F.; TEIXEIRA, F.A.T. Análise sistêmica da fruticultura paraense: organização, mercado e competitividade empresarial. Belém: Banco da Amazônia, 2008. $255 \mathrm{p}$.

SMITH, H.F. A discriminant function for plant selection. Annual of Human Genetics, v.7, p.240-250, 1936.

STEEL, R.G.D.; TORRIE, J.H.; DICKEY, D.A. Principles and procedures of statistics: a biometrical approach. New York: McGraw Hill, 1997. 672p.

WILLIAMS, J.S. The evaluation of a selection index. Biometrics, v.18, p.375-393, 1962.

Recebido em 1ํ de setembro de 2011 e aprovado em 9 de janeiro de 2012 